\title{
Calvillo Castro, A.J.(Coord.) (2019). Un mundo de proyectos ABP musicales (2 vol.). Almería: Procompal
}

El aprendizaje basado en proyectos (ABP) es un método que supone "una búsqueda de nuevos modos de hacer, aprender y enseñar" (Moya-Mata y Peirats, 2019, p.115) que, aunque comenzó usándose en las etapas de estudios superiores, ha demostrado buenos resultados en su aplicación en enseñanzas obligatorias (Molina, 2018).

Complementar nuestra metodología habitual con el desarrollo de un ABP aporta al alumnado el aumento de la motivación, la mejora del autoaprendizaje y comprensión, el desarrollo del pensamiento y el aumento de la retención (Rodríguez, Fernández y Hernández, 2018). El ABP es una metodología en la que el alumnado asume un papel principal en el desarrollo del producto final (del Moral y Sobrino, 2016). Fernando Trujillo (2014) afirma que "el aprendizaje basado en proyectos, lejos de ser una extravagancia es una metodología que encaja perfectamente en la LOE y su modificación por la LOMCE" (p.44), motivo por el que se convierte en el aliado perfecto de la educación musical a todos los niveles.

El libro Un mundo de proyectos ABP musicales presenta 37 proyectos musicales realizados por 25 docentes de todas las etapas de nuestro sistema educativo. La obra, estructurada en dos volúmenes, recoge proyectos desde Educación Infantil hasta Educación Superior. En ella, el docente puede encontrar material sobre fundamentos musicales, participación de la comunidad, juego, gamificación, investigación-acción, aplicaciones tic para el aula de Música o metodologías activas varias, entre otros temas.

Como menciona Miguel Domínguez (2019), "el contenido visual posee un gran impacto y, adecuadamente utilizado, tiene un alto valor didáctico" (p.421), hecho que en esta obra se desarrolla ampliamente, ya que los libros están ilustrados con la técnica Visual Thinking, por $\mathrm{M}^{\mathrm{a}}$ Pilar Muruzábal. De esta manera, el profesorado puede conocer el contenido de los casi cuarenta proyectos que se presentan de una manera novedosa, visual y rápida.

En el prólogo, elaborado por la Dra. Andrea Giráldez, se menciona la necesidad de crear los estímulos que generen procesos de enseñanza y aprendizaje; de manera que se anime al alumnado a participar en la educación musical, principalmente, en las etapas intermedias, ya que es cuando se detecta cierto rechazo a la misma. El ABP ha demostrado ser un recurso valioso en este aspecto. A lo largo de la introducción, el Dr. Antonio J. Calvillo, coordinador de la obra, explica los beneficios que esta metodología aporta al alumnado.

En el primer volumen, pueden encontrarse los cinco proyectos que se proponen para Educación Infantil. El profesorado encargado de presentar los proyectos destinados a 3-6 años está formado por Lidia González, Patricia Huertas, Soledad Mellada y Jesús Sáez. Se puede

@Mauricio Rodríguez López y Antonio Jesús Calvillo Castro. The content of this article is the sole responsibility of the authors. The Revista Electrónica de LEEME and Universitat de València are not liable for any legal actions that may arise involving the article's content. Revista Electrónica de LEEME - Lista Electrónica Europea de Música en la Educación-. http://ojs.uv.es/index/php/LEEME/index ISSN: 1575-9563. Editores: Universidad de Valencia y Jesús Tejada. Visibilidad de esta revista: SCOPUS, Emerging Sources Citation Index (Clarivate), EBSCO, CINDOC (CSIC), Citefactor, COPAC, Dialnet, DICE (CSIC), DOAJ, e-revistas (CSIC), EBSCO Premier, ERIH+, Gale Cengage Learning, IN-RECS, IRESIE, LATINDEX, MIAR, OCLC Worldcat, RESH, REDIB, RILM Core Journals, SUDOC, ULRICHS. Esta revista es de acceso libre mediante licencia Creative Commons 4.0 CC by. Política de archivado: etiqueta verde SHERPA-ROMEO. 

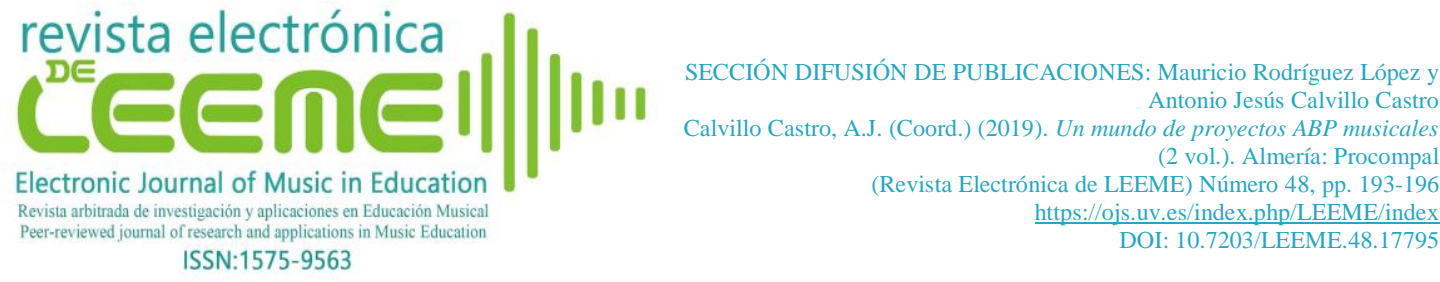

destacar el proyecto Operación: cómo suena mi clase, de J. Sáez. Este ABP tiene como objetivo estimular el sistema auditivo verificando que el aprendizaje del lenguaje es el correcto, asociando nuevos sonidos a nuevas palabras, proceso fundamental en la etapa de Educación Infantil. El desarrollo de este proyecto hará que el alumnado profundice en el tema desde la gamificación, pero sin dejar atrás los fundamentos musicales.

Cada proyecto ha situado un escenario inicial, un camino de aprendizaje para el alumnado con tareas abiertas y motivadoras que desembocan en la realización de uno o varios productos finales. De este modo, se integran los conocimientos adquiridos por el alumnado y que son el ejemplo de su propio aprendizaje o la solución al problema planteado.

Se completa el primer volumen con la propuesta para Educación Primaria, formada por diez proyectos que abordan los instrumentos de la orquesta: La orquesta trotamundos de Natalia Barreno; las compositoras de la historia de la música con Los juegos de género, creado por Carmen Carvajal; ;Berceo al rescate!, escrito por $\mathrm{M}^{\mathrm{a}}$ Pilar Muruzábal, en el que se trabaja la historia, programación con scratch y aplicación al aula con makey makey; o Suéname un cuento de Lidia González, en el que las áreas de música, lengua y plástica se fusionan para conectar aprendizajes.

En el volumen dos, pueden encontrarse hasta 22 proyectos desarrollados en Educación Secundaria, Formación Profesional Básica, Bachiller y Educación Superior. Estos proyectos muestran los productos finales y procesos seguidos por catorce docentes del área de música. En los capítulos dedicados a la ESO, se viaja de la ópera romántica al rap pasando por la realidad aumentada, el reciclaje, la cultura celta o los paisajes de aprendizaje con el proyecto La invasión zombie, desarrollado por la profesora Ana $\mathrm{M}^{\mathrm{a}}$ Martínez.

El ABP Este es mi barrio, creado por Blanca Domínguez, guía al lector/a paso a paso hasta desarrollar una batucada muy especial en la que el alumnado deberá construir sus propios instrumentos de percusión. Este proyecto se puede completar ejecutando los ritmos latinos aprendidos en clase en las calles cercanas al centro educativo, a modo de perfomance. En la misma línea de construir instrumentos, se encuentra el proyecto Los Luthiers del vertedero, creado por Zoraida Pérez, aunque en esta ocasión el proyecto abarca todas las familias de instrumentos y se desarrolla de manera interdisciplinar con la ayuda de las áreas de Matemáticas y Tecnología, con la intención final de crear la orquesta del reciclaje.

Uno de los aspectos más interesantes de la obra es que recoge todas las etapas educativas mostrando cómo en la Educación Superior también se puede aprender usando la metodología del ABP al igual que se hace en Educación Infantil. En concreto, se presentan cuatro proyectos independientes desarrollados en las universidades de Cantabria, Segovia, Valladolid y Almería. Estos proyectos son independientes, pero mantienen un objetivo común:

@ Mauricio Rodríguez López y Antonio Jesús Calvillo Castro. The content of this article is the sole responsibility of the authors. The Revista Electrónica de LEEME and Universitat de València are not liable for any legal actions that may arise involving the article's content. Revista Electrónica de LEEME - Lista Electrónica Europea de Música en la Educación-. http://ojs.uv.es/index/php/LEEME/index ISSN: 1575-9563. Editores: Universidad de Valencia y Jesús Tejada. Visibilidad de esta revista: SCOPUS, Emerging Sources Citation Index (Clarivate), EBSCO, CINDOC (CSIC), Citefactor, COPAC, Dialnet, DICE (CSIC), DOAJ, e-revistas (CSIC), EBSCO Premier, ERIH+, Gale Cengage Learning, IN-RECS, IRESIE, LATINDEX, MIAR, OCLC Worldcat, RESH, REDIB, RILM Core Journals, SUDOC, ULRICHS. Esta revista es de acceso libre mediante licencia Creative Commons 4.0 CC by. Política de archivado: etiqueta verde SHERPA-ROMEO. 

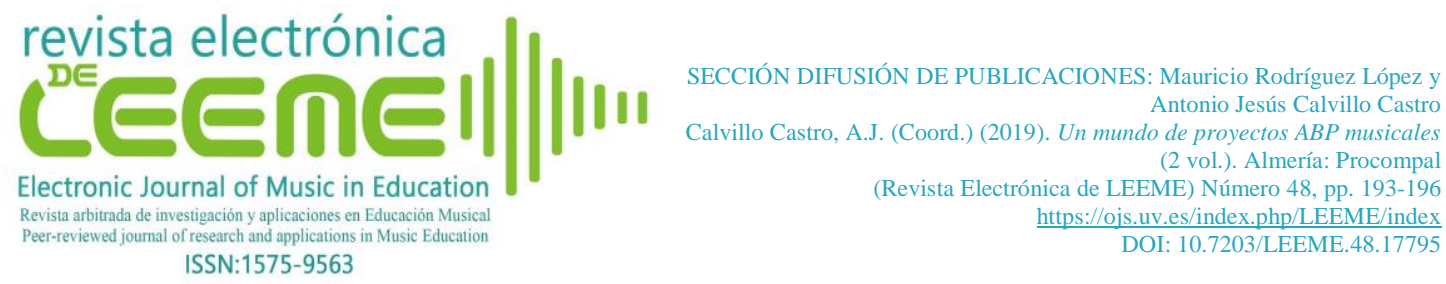

hacer que el alumnado de Grado en Educación se convierta en el protagonista de su aprendizaje, y lo haga desde la reflexión crítica sobre los propios métodos y materiales.

Las autoras y autores del libro han aportado a cada proyecto todo el material desarrollado en el aula, no solo ideas o posibles tareas, con lo cual su aplicación ya parte del éxito conseguido en otros centros. Como señala la Dra. Andrea Giráldez en el prólogo, no se trata de una fórmula mágica que podamos replicar, sino de un material didáctico que facilitará la tarea de crear nuestro ABP.

El/la lector/a encontrará recursos para trabajar la Realidad Aumentada, impresión en 3D, robótica, desarrollo de la gamificación, aplicación de paisajes de aprendizaje, completos breakout y Escaperoom, además de la necesaria práctica instrumental y vocal, composición y creatividad, danza y teatro. Aunque el eje de todos los ABP sea la música, se pueden encontrar productos finales relacionados con Historia, igualdad de género o nuevas tecnologías.

El libro está ilustrado con la mencionada técnica Visual Thinking para agregar un valor añadido, animando al docente a introducir la creatividad en su aula con la intención de generar un cambio en su método de enseñanza. Además de las figuras de Visual Thinking, cada proyecto se encuentra ilustrado ejemplificando los procesos seguidos y los productos conseguidos. Los $\mathrm{ABP}$ se completan con enlaces y códigos QR que facilitan el acceso a tareas, vídeos, material complementario, productos finales y ejemplificaciones reales de cada uno de ellos. Todos los proyectos presentan un apartado de conclusiones que reflexionan sobre el proceso, las ventajas y los problemas detectados durante el desarrollo de los diversos pasos del ABP, aportando sugerencias de mejora y ayudando así al lector a su adaptación y desarrollo.

Trabajar en el aula bajo el ABP consigue que el alumnado desarrolle su habilidad, creatividad y competencias a la vez que se fomenta el trabajo cooperativo y se activa la interdisciplinariedad. En el número 42 de la Revista Electrónica de LEEME, Yelo (2018) ya dejaba claro que "el desarrollo de la creatividad debería ser una de las funciones de la escuela, no sólo en el estudiantado, sino también en los docentes" (p.87). En su experiencia desarrollando creatividad en el aula de Música, concluía que "es importante valorar el proceso más que el resultado" (p.95).

La lectura de los dos volúmenes aportará los recursos necesarios para enfocar el trabajo del aula desde una perspectiva activa y participativa, persiguiendo la necesaria motivación del alumnado. Es una obra recomendable para docentes de cualquier área por los aportes didácticos que se han presentado, aunque haya sido creada por especialistas en educación musical. No se trata de un manual didáctico que el profesorado deba aplicar a su aula, sino un compendio de productos finales con sus correspondientes orientaciones y materiales para que puedan adaptarlos a la realidad de su centro. Así pues, todos los proyectos presentados pueden ser

@ Mauricio Rodríguez López y Antonio Jesús Calvillo Castro. The content of this article is the sole responsibility of the authors. The Revista Electrónica de LEEME and Universitat de València are not liable for any legal actions that may arise involving the article's content. Revista Electrónica de LEEME - Lista Electrónica Europea de Música en la Educación-. http://ojs.uv.es/index/php/LEEME/index ISSN: 1575-9563. Editores: Universidad de Valencia y Jesús Tejada. Visibilidad de esta revista: SCOPUS, Emerging Sources Citation Index (Clarivate), EBSCO, CINDOC (CSIC), Citefactor, COPAC, Dialnet, DICE (CSIC), DOAJ, e-revistas (CSIC), EBSCO Premier, ERIH+, Gale Cengage Learning, IN-RECS, IRESIE, LATINDEX, MIAR, OCLC Worldcat, RESH, REDIB, RILM Core Journals, SUDOC, ULRICHS. Esta revista es de acceso libre mediante licencia Creative Commons 4.0 CC by. Política de archivado: etiqueta verde SHERPA-ROMEO. 

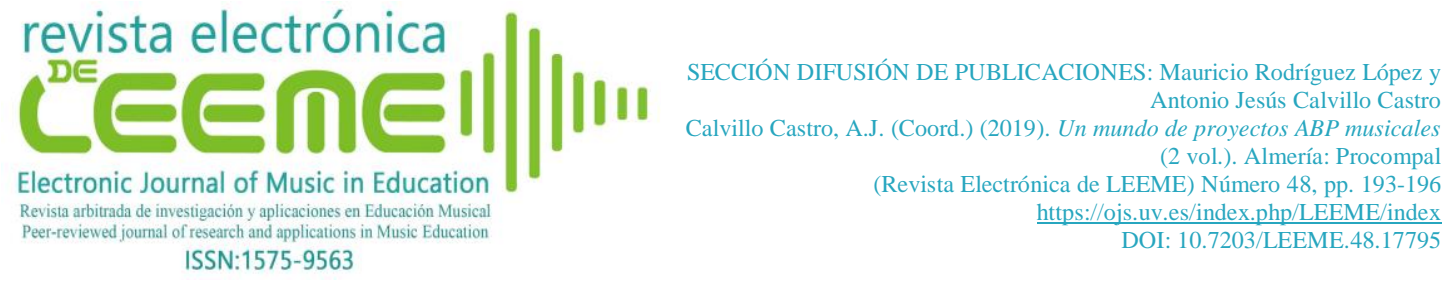

ajustados independientemente de la etapa en la que se hubieran desarrollado, sin importar si somos o no especialistas de Música.

\section{Referencias}

del Moral, C. y Sobrino, D. (2016). Aprendizaje basado en proyectos en Ciencias Sociales. Revista Iber, 82, 4-6. https://www.grao.com/es/producto/

Domínguez, M. (2019). Visual thinking y creatividad. En M. Larragueta, (coord.), Educación y transformación social y cultural (pp.421-426). Editorial Universitas.

Trujillo, F. (2014). Aprendizaje basado en proyectos: infantil, primaria y secundaria. Ministerio de Educación y Formación Profesional.

Yelo, J.J. (2018). La recreación artística de textos e imágenes como modelo para el desarrollo de la creatividad y la integración de los lenguajes expresivos en el aula de música. Revista Electrónica de LEEME, 42, 84-98. https://doi.org/10.7203/LEEME.42.13171

Moya-Mata, I. y Peirats, J. (2019). Aprendizaje basado en Proyectos en Educación Física en Primaria, un estudio de revisión. REIDOCREA, 8(2), 115-130. http://www.ugr.es/ reidocrea/

Molina, $M^{\mathrm{a}}$.P. (2018). Aprendizaje basado en proyectos (ABP) para la enseñanza de la historia. En A.I. Ponce y J. Ortuño Molina (Eds.), Usos y recursos en el ámbito educativo (pp.127-138). Editorial Universidad de Murcia.

Rodríguez, M., Fernández y P. Hernández, P. (2018). Realidad aumentada y ABP como recursos para mejorar el rendimiento académico. En E.J. López-Meneses (Dir.), Experiencias pedagógicas e innovación educativa. Aportaciones desde la praxis docente e investigadora (pp.231-246). Editorial Octaedro.

Dr. Mauricio Rodríguez López. Profesor de Didáctica de la Música en Facultad de Educación, Universidad de Almería maurirrr@ual.es Dr. Antonio Jesús Calvillo Castro. Profesor de Música en el IES Doñana de Sanlúcar de Barrameda (Cádiz) caotico27@gmail.com

@ Mauricio Rodríguez López y Antonio Jesús Calvillo Castro. The content of this article is the sole responsibility of the authors. The Revista Electrónica de LEEME and Universitat de València are not liable for any legal actions that may arise involving the article's content. Revista Electrónica de LEEME - Lista Electrónica Europea de Música en la Educación-. http://ojs.uv.es/index/php/LEEME/index ISSN: 1575-9563. Editores: Universidad de Valencia y Jesús Tejada. Visibilidad de esta revista: SCOPUS, Emerging Sources Citation Index (Clarivate), EBSCO, CINDOC (CSIC), Citefactor, COPAC, Dialnet, DICE (CSIC), DOAJ, e-revistas (CSIC), EBSCO Premier, ERIH+, Gale Cengage Learning, IN-RECS, IRESIE, LATINDEX, MIAR, OCLC Worldcat, RESH, REDIB, RILM Core Journals, SUDOC, ULRICHS. Esta revista es de acceso libre mediante licencia Creative Commons 4.0 CC by. Política de archivado: etiqueta verde SHERPA-ROMEO. 